\title{
Using finite element analysis to obtain plastic zones in the vicinity of the crack edges, under mixed mode loading conditions
}

\author{
Matúš Turis ${ }^{1, *}$, Ol'ga Ivánková ${ }^{2}$ \\ ${ }^{1}$ Slovak University of Technology in Bratislava, Faculty of Civil Engineering, Department of \\ Structural Mechanics. 81005 Bratislava, Slovak Republic \\ ${ }^{2}$ Slovak University of Technology in Bratislava, Faculty of Civil Engineering, Department of \\ Structural Mechanics, 81005 Bratislava, Slovak Republic
}

\begin{abstract}
This paper deals with both size and shape of plastic zone in the vicinity of the crack edges. Determination of plastic zone is proposed using the three-dimensional finite element analysis. Both, linear elastic and elastic-plastic material models are considered in this paper. Subsequently, results of these approaches are compared and their applicability is evaluated. Geometry considered is a beam with L-shaped cross section containing a crack, as it is described below. Mentioned beam is subjected to mixed mode loading condition, that is represented by variable forces in the direction of global coordinate system. Evolution of plastic zones is observed and evaluated around the crack edges of the profile.
\end{abstract}

\section{Introduction}

At the production stage of structural elements, various material errors and cracks arise, that is natural part of this process. Main goal of fracture mechanics is to understand the components behavior with these disturbances to ensure their safety and usability. Therefore, it is very important to describe the behavior of structures in real conditions with the presence of various defects or cracks in materials.

Considering the linear elastic material, the stress analysis in the vicinity of the crack tip, leads to a stress singularity at $r \rightarrow 0$ where the stress tensor components $\sigma_{i j} \rightarrow \infty$. Materials, that exhibit plastic deformation exceed the yield point at the stress concentration area resulting in local plastic deformation. In the plastic zone around the crack tip, the stress will be largest, but its amplitude will be limited by the material stress-strain curve. For this reason, it is also necessary to consider the plastic deformations, when evaluating crack stability.

This article will gradually describe the basic principles in the determination of the plastic zones around the crack tip. The problem is solved as a three-dimensional task using the finite element method. We considered mixed mode loading of crack and the obtained results are consequently evaluated.

\footnotetext{
*Corresponding author: matus.turis@stuba.sk
} 


\section{Boundary condition of solved problem}

Considered geometry, shown in Fig. 1., is a beam with L-shaped cross section containing a corner crack. Main dimensions are the length of profile $\mathrm{L}=200 \mathrm{~mm}$, profile web height and width $w=20 \mathrm{~mm}$, thickness of webs $b=1.5 \mathrm{~mm}$ and considered crack length $\mathrm{a}=5 \mathrm{~mm}$.

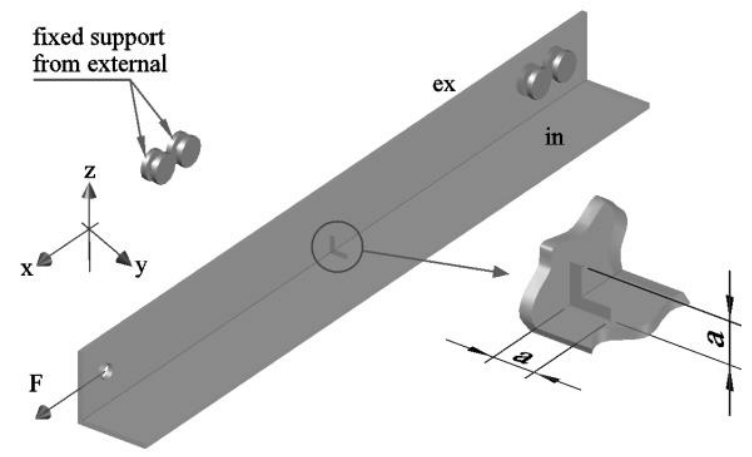

Fig. 1. Geometry of cracked body and global coordinate system.

The profile is at one side attached by pair of bolts, that have fixed support from external side. Other side is loaded with force in several variants of action. Forces in directions of global coordinates $\mathrm{y}, \mathrm{z}$ is considered in five scenarios with respect to ratio to the constant force acting in the direction of longitudinal axis $\mathrm{x}$.

\section{Defining the concepts of fracture mechanics}

Using the linear elastic fracture mechanics (LEFM) principles, the solution of stress field in the vicinity of the crack tip can be described using one single parameter, the stress intensity factor (SIF). There are three different SIFs due to the loading condition of crack, i.e. loading modes, shown in Fig. 2.

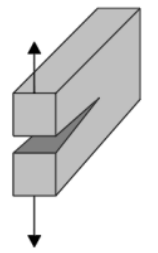

(a)

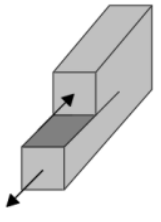

(b)

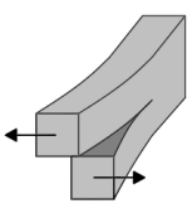

(c)

Fig. 2. The three basic loading modes of crack (a) Mode I (b) Mode II (c) Mode III.

Each of this loading modes contain a singular component represented by $r^{-0.5}$. Using known SIFs, the stresses in the vicinity of crack edge can by calculated using polar coordinates $r$ and $\theta$, shown in Fig.3. 


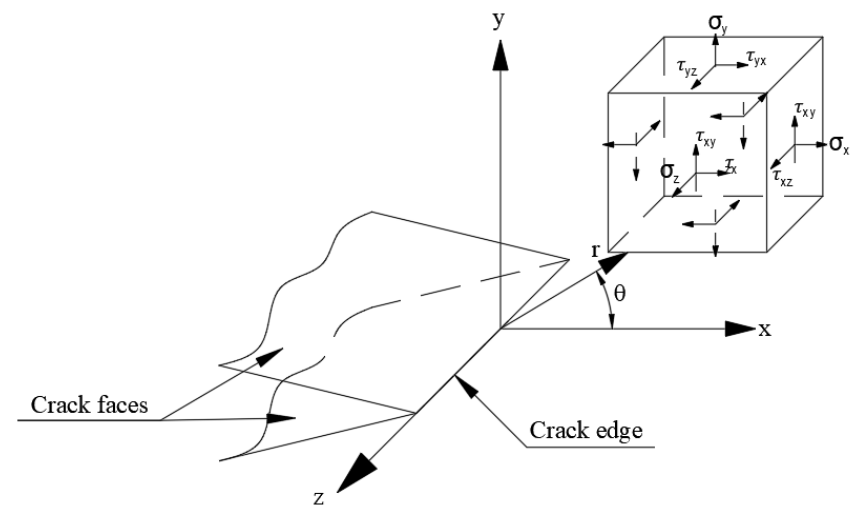

Fig. 3. The stress tensors and local coordinate system of the crack.

In case when more than one loading mode occurred, the final solution of stress fields can be described using the principles of superposition. For further information see the handbooks dealing with fracture mechanics problems, e.g. [1], [2], [3].

Considering materials capable of plastic deformation, the plasticity can occur around the crack edge. As a result, the stresses are bounded by the stress-strain curve of the material and the singularity problem disappears.

First approximation of the size and shape of plastic zone can be obtained from von Misses yield criterion. The equivalent stresses (defined by von Mises) can be obtained by individual components of stress tensor. Based on this criterion, plastic zone shall occur in the location, where the equivalent stress exceeds the yield point. Boundaries of yield stress obtained by von Mises criteria for plane strain condition are shown in Fig.4, where $r_{y s}$ is a polar coordinate of yield area boundary and $\sigma_{y s}$ is a yield stress. Note that $K_{I}$ value is set to be constant and $K_{I I}$ and $K_{I I I}$ are variables.

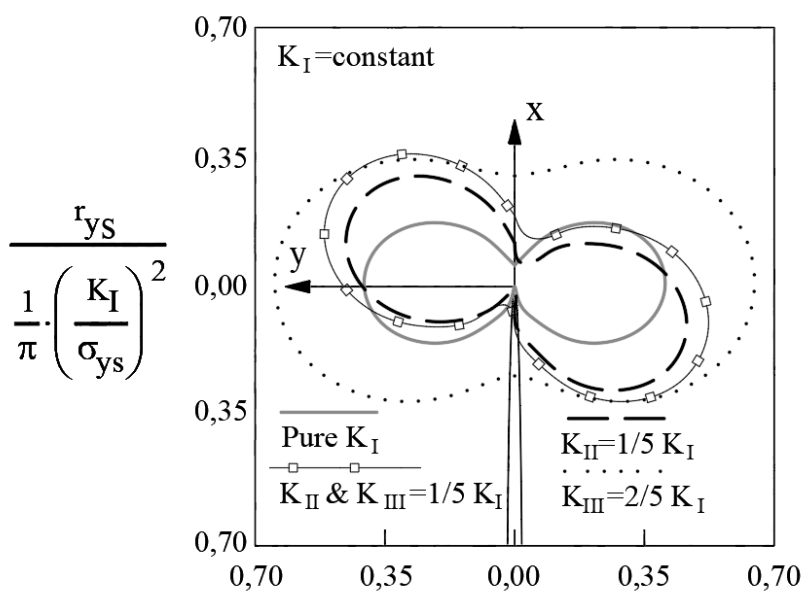

Fig. 4. Isolines of plastic area boundaries for mixed mode of loading, where $K_{I}$ value is constant and $K_{I I}, K_{I I I}$ are variables.

This approach provides an approximate estimate of the size and shape of the plastic zone around the crack tip. To obtain real size and shape of plastic zone for nonlinear materials, it is necessary to use experimental or numerical analysis. 


\section{Numerical analysis}

Numerical analysis was conducted by the Ansys software, using the finite element method. The analysis itself was divided in several steps. At first SIFs was calculated for various loading condition. Subsequently, corresponding yielded volumes, based on the principle of the von Mises criterion were determined. Finally, the analysis considering the elastic-plastic material was completed.

\subsection{Determination of SIF}

First step of numerical analysis was the determination of SIFs for each scenario of considered problem. Individual SIFs was calculated using the node displacement extrapolation method as mentioned in $[4,5]$.

Using this method, it was possible to calculate SIF directly from displacements on the so-called singular elements around the crack edge. This type of element was first presented in [6]. In our case the singular element was achieved by degenerating the Solid 186 to the shape of prism and mid nodes were shifted to a quarter of element length.

\subsection{Analysis of elastic-plastic material}

Based on the calculation of plastic zone size for linear elastic material, the finite element mesh was refined in the area of predicted plastic deformation. Outside of this zone, there was a transition area of mesh to elements with larger dimensions. The used mesh was primarily hexahedral, except the location in the vicinity of the crack edge, as mentioned above. Material with strain-hardening rate $n=50$, that characterizing low strain-hardening, was considered according to [7].

\section{Results and discussion}

Results showed bellow were verified with previous study of plastic zones on the single edge notch bending specimen. The aim of the study was to estimate the plastic zone in the condition of plane stress, plane strain, and considering the three-dimensional problem. These results were compared with [1], [8-10].

Results of so called "preparation analysis" can be summarized into evaluation of plastic volume for pure mode $I$ loading condition as showed in Fig. 5.

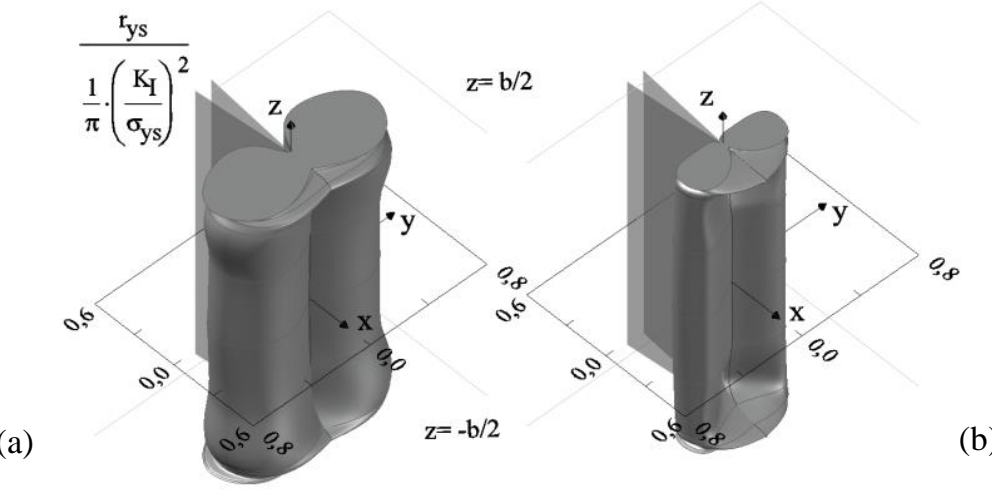

Fig. 5. Yielded volume for pure mode $I$ loading obtained by numerical analysis for (a) linear elastic material (b) material with strain-hardening rate $n=50$. 
Theoretical yielded volume when considering only linear elastic material was approximately three times larger, than in the case when considering material is capable to plastic deformation (in Fig. 5).

\subsection{Linear-elastic fracture mechanics approach}

As stated above, SIFs in this article are determined using the node displacement extrapolation method. This method is very effective tool for determining SIFs. The main advantage of this method instead the interaction integral method is saving the computational time. SIFs are computed directly from displacements of nodes placed on the singular elements mentioned above. Results of individual SIFs are shown in Fig. 6. These results are dimensionless as achieved by dividing actual SIF with $K_{\infty}=\sigma(\pi a)^{0.5}$, where $\sigma$ is applied force in the $\mathrm{x}$ direction divided by cross sectional area of profile.

Crack edge on the top web of profile
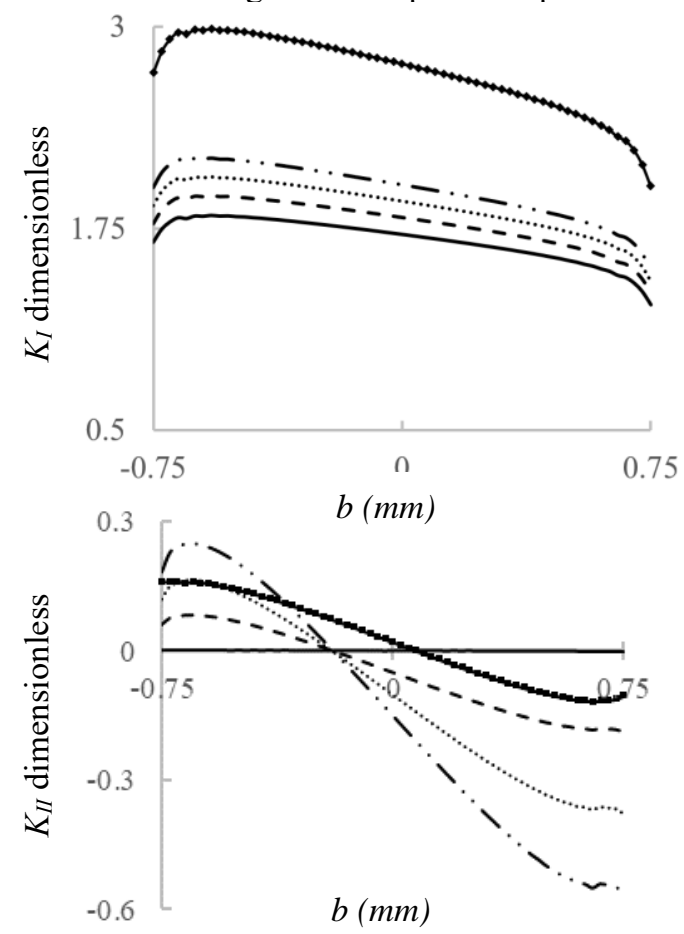

Crack edge on the bottom web of profile
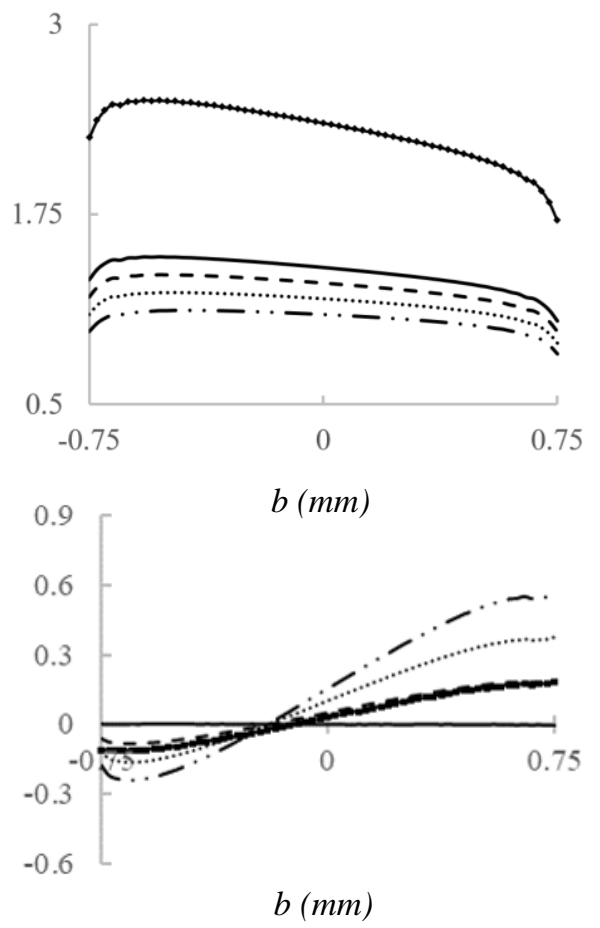

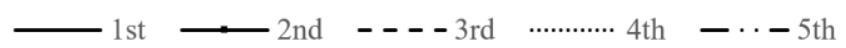

Fig. 6. Through thickness variation of SIFs, where negative value of $b$ means external side of profile (the sequel is on the next page). 


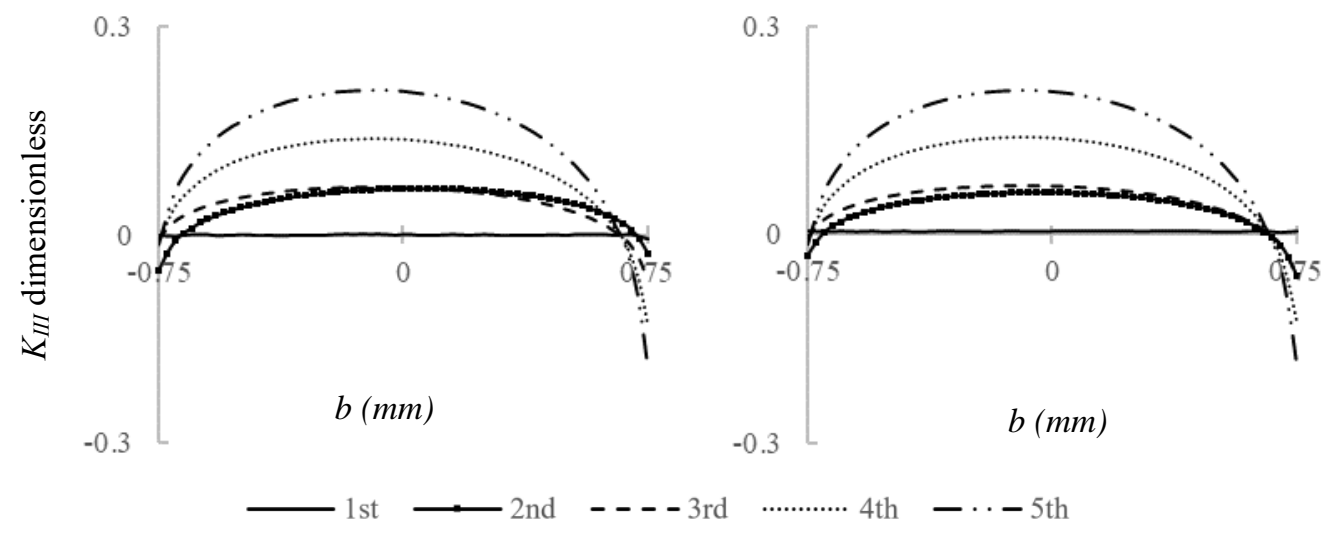

Fig.6. Through thickness variation of SIFs, where negative value of $b$ means external side of profile.

The force in longitudinal axis $\mathrm{x}$ is still a present constant and forces in direction $\mathrm{y}, \mathrm{z}$ are variables. Individual marking numbers in Fig. 6 represents: 1st $\mathrm{F}_{\mathrm{y}}=0 \mathrm{~F}_{\mathrm{z}}=0 ; 2 \mathrm{nd} \mathrm{F}_{\mathrm{y}}=\mathrm{F}_{\mathrm{x}} \cdot 30^{-1}$ $\mathrm{F}_{\mathrm{z}}=0 ; 3 \mathrm{rd} \mathrm{F}_{\mathrm{y}}=\mathrm{F}_{\mathrm{x}} \cdot 30^{-1} \mathrm{~F}_{\mathrm{z}}=-\mathrm{F}_{\mathrm{x}} \cdot 30^{-1} ;$ th $\mathrm{F}_{\mathrm{y}}=\mathrm{F}_{\mathrm{x}} \cdot 15^{-1} \mathrm{~F}_{\mathrm{z}}=-\mathrm{F}_{\mathrm{x}} \cdot 15^{-1} ;$ th $\mathrm{F}_{\mathrm{y}}=\mathrm{F}_{\mathrm{x}} \cdot 10^{-1} \mathrm{~F}_{\mathrm{z}}=-\mathrm{F}_{\mathrm{x}} \cdot 10^{-1}$.

The theoretical yielded volumes with respect to Fig. 6 are shown in Fig. 7. where ratios of actual yielded volume to yielded volume occurred when applying only axial force are graphically evaluated.

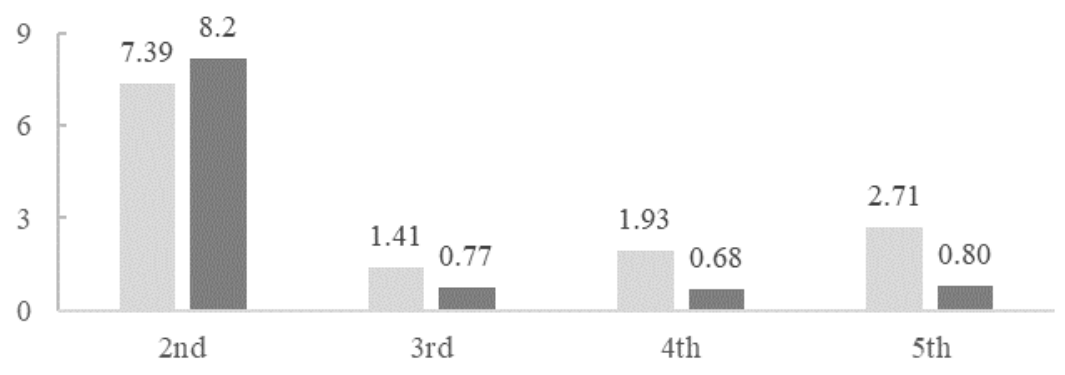

Top web location of crack edge $\quad$ Bottom web location of crack edge

Fig. 7. Theoretical values of yielded volumes ratio.

Using theoretical means, that these volumes are obtained only from linear elastic model. It can be seen, that maximum theoretical value of yielded volume ratio occurred at scenario 2, see Fig.7. At the same time this scenario reached maximum values of $K_{I}$, that was much higher value, than the other two SIFs, see Fig. 6. For this reason, force in the $\mathrm{z}$ direction is applied to cause the closure effect of crack so that each loading modes are comparable.

\subsection{Elastic-plastic analysis}

In this chapter results for the fifth scenario of loading crack are evaluated. Forces in the perpendicular direction are considered as $10 \%$ of the axial force. In Fig. 6 the chart of SIFs for this type of loading condition is depicted with two dotted and one dashed line.

Results of plastic zone shape for the crack edge located on the bottom web of the profile are shown in Fig.8. Depicted coordinate system in Fig.8 is a local coordinate system of the crack, showed in Fig.3. 


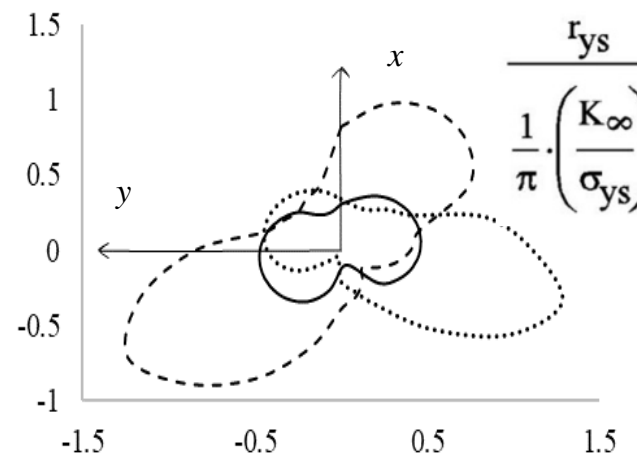

(a)

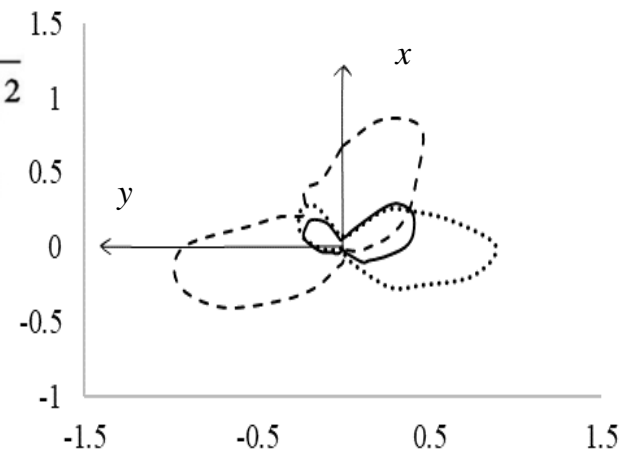

(b)

bott_ex - bott_mid - - - bott_in

Fig. 8. Plastic zone shape for bottom edge of crack in three through body thickness position for (a) LEFM approach (b) elastic-plastic analysis.

Results for crack edge located on the top web of the profile are graphically evaluated in Fig. 9, where the three-dimensional shape of plastic zone is shown. Coordinate system shown od Fig. 9 is a local coordinate system of crack.

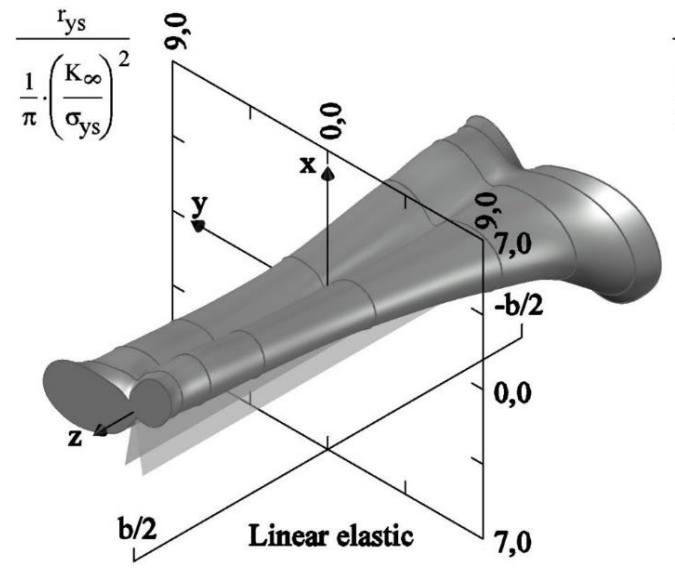

(a)

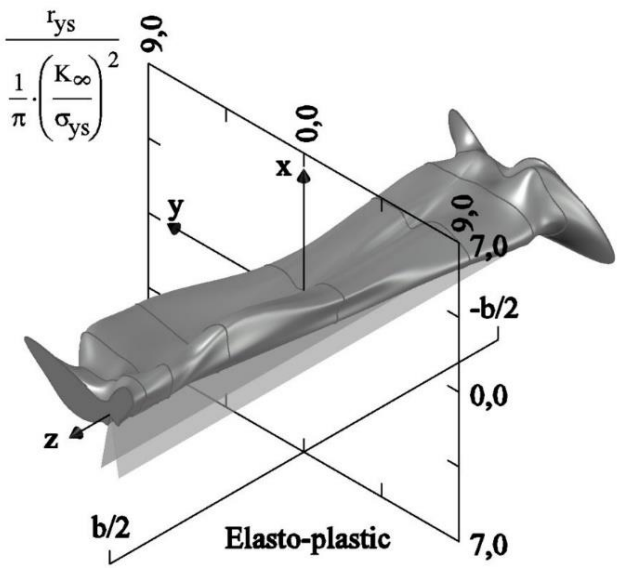

(b)

Fig.9. Plastic zone shape around the crack edge located on the top web of profile for (a) LEFM approach (b) elastic-plastic analysis.

\section{Conclusion}

In this paper the determination of plastic zones around the crack edges was carried out. It was pointed out, that for different loading conditions, the yielded volume changed its shape and size. Each loading conditions were represented by ratio of variable forces in global coordinate system $\mathrm{y}, \mathrm{z}$ to axial force, that was acting in the longitudinal direction of profile.

On the given geometry, only relatively small forces perpendicular to longitudinal axis of profile to initiated mixed mode loading effects were needed. This mixed mode loading conditions affected the plastic zone size and generally affected the stability of crack itself. 
Due to this fact, it is needed to consider every possible loading cases of a body including a crack. Presented results needs to be experimentally verified.

This article was written thanks to the grant program SK-VEGA 1/0412/18 and SK-KEGA 025 STU$4 / 2019$.

\section{References}

1. T.L. Anderson, Fracture mechanics Fundamentals and Applications, 688s, 3 (1995)

2. F. Bahram, Mh. Aliabadi, Fracture Mechanics of Metals, Composites, Welds, and Bolted Joints: Application of LEFM, EPFM, and FMDM Theory. (2002)

3. J. Kunz, Aplikovaná lomová mechanika. 4. (1991)

4. A. Boulenouar, N. Benseddiq, M. Mazari, ETASR - Engineering, Technology \& Applied Science Research, Vol. 3, pp. 506-510, (2013)

5. M. Nejati, A. Paluszny, R. Zimmerman, Engineering Fracture Mechanics, 133, pp.140 (2015)

6. R.S. Barsoum, International Journal for Numerical Methods in Engineering, 10, pp. 2537, (1976)

7. R. H. Dodds Jr., T.L. Anderson, M.T. Kirk, International Journal of Fracture, 48, pp.122, (1991)

8. K. Shashidhar, K. Krishnaraja, International Journal of Engineering, Science and Technology, 2, pp. 47-58 (2010)

9. N. Ramarathnam, H. Subramanya, P. Swapnil, T. Parag, U. Ramamurty, Journal of Physics D: Applied Physics, 42, (2009)

10. D. Camas, I. Hiraldo, P. Lopez-Crespo, A. Gonzalez-Herrera, Procedia Engineering, 10, pp. 1691-1696 (2011) 